\title{
sciendo
}

Current Issues in Pharmacy and Medical Sciences

Formerly ANNALES UNIVERSITATIS MARIAE CURIE-SKLODOWSKA, SECTIO DDD, PHARMACIA

journal homepage: http://www.curipms.umlub.pl/

\section{Optimization of the composition of solid dispersion of quercetin}

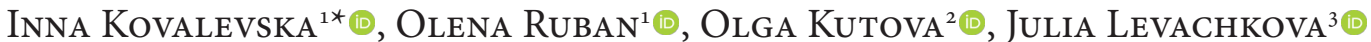

\author{
${ }^{1}$ Department of Industrial Technology of Drugs, National University of Pharmacy, Kharkov, Ukraine \\ ${ }^{2}$ Department of Processes and Equipment of Chemical and Pharmaceutical Production, National University of Pharmacy, Kharkov, \\ Ukraine \\ ${ }^{3}$ Department of Drug Technology, National University of Pharmacy, Kharkov, Ukrain
}

\begin{tabular}{l}
\hline ARTICLE INFO \\
\hline Received 07 October 2019 \\
Accepted 08 December 2020
\end{tabular}

Keywords:

polyethyleneoxide-6000,

$\beta$-cyclodextrin,

polyvinylpyrrolidone,

mathematical planning

method,

quercetin,

solid dispersions.

\begin{abstract}
Increasing the solubility of drugs is one of the most important problems in pharmacy, because most manufactured pharmaceutical substances are sparingly soluble. Typically, such compounds represent Classes II and IV in the biopharmaceutical classification system, which is based on the classification of pharmaceutical substances by solubility and permeability. For poorly soluble substances, the limiting step of the absorption process is usually the degree and dissolution rate, so in the pharmaceutical development, much attention is paid to their improvement. To solve this problem, the technology of solid dispersions creation is used. The aim of the work was to optimize the composition of quercetin solid dispersion, whose therapeutic use is limited due to its degree of solubility in an aqueous medium.

Objects of the study were samples of solid dispersions of quercetin with different carriers: polyethyleneoxide-6000, $\beta$-cyclodextrin and polyvinylpyrrolidone obtained in a ratio of 1:1 and 1:2 by liquid phase method, with and without the addition of a solvent. As a solvent, ethanol was used. To determine the optimal composition of solid dispersions, a mathematical planning method using the MathCad package and Excel software was used.

In the work, a dispersion analysis was performed upon the results of the experimental studies. The ranked series of advantages of the carrier and solvent influence on solubility of solid dispersions samples are shown. To determine the amount of carrier in the solid dispersion, the theory of multi-vector optimization was applied. According to the results of the conducted studies, the optimal value of quercetin and PEO-6000 ratio is 1:2.

The obtained results can be used in the development of the composition and technology of solid dispersions of active pharmaceutical ingredients insoluble in the aqueous medium.
\end{abstract}

\section{INTRODUCTION}

In diabetes mellitus, the balance between the processes of oxidation and antioxidant defense is disturbed. A high level of sugar in the blood leads to the activation of oxidative stress and provokes the occurrence of complications. The use of antioxidant therapy in diabetes can prevent or slow down the development of the disease and its complications [1]. One of the most commonly used substances in diabetes with antioxidant properties is quercetin. Its antioxidant action is conditioned by the ability to inactivate free radicals that are formed as a result of peroxide oxidation.

\footnotetext{
* Corresponding author

e-mail: inga.kovalevskaya@gmail.com
}

The blocking of free radical lipoperoxidation is due not only to the structural features of the substance, but also to its ability to interact with the membranes and penetrate through their lipid layer. According to literature, it is known that quercetin also exhibits hypoglycemic activity as it stimulates the release of insulin [2].

Previous studies have found that quercetin is practically insoluble in an aqueous medium, which significantly impairs its bioavailability [3]. To improve the solubility, the technology of solid dispersions creation is used, which allows increasing substance solubility by changing their physical and chemical properties [4]. 
To date, to study the simultaneous effect of a large number of variables on the characteristics of the finished product, it is recommended to use the method of experimental mathematical planning. This allows minimizing the number of experiments and selecting the parameters of the process - which will lead to the obtaining of preparations with the necessary properties.

Thus, the aim of the work was to optimize the composition of quercetin solid dispersion.

\section{MATERIALS AND METHODS}

The objects of the study were specimens of solid dispersions of quercetin (Q) with different carriers: polyethylene oxide-6000 (PEO-6000) (sample 1), $\beta$-cyclodextrin ( $\beta$-CD) (sample 2), polyvinylpyrrolidone (PVP) (sample 3) obtained in a ratio of $1: 1$ and $1: 2$ by liquid phase methods [3]. Samples were prepared as follows: 1 - quercetin was dissolved in ethanol, mixed with molten PEO-6000 ( $\mathrm{T}=55$ 60C) and dried, 2, 3-quercetin was dissolved in ethanol, mixed with the carrier and dried. Determination of disintegration, bulk density, fluidity, crushing strength, abrasion resistance and dissolution time was performed according to the requirements of the $\mathrm{Ph}$. Eur. Model specimens of tablets were obtained on a laboratory single-punch eccentric tablet press (matrix diameter - $11 \mathrm{~mm}$, flat-faced punches, compression pressure - $120 \mathrm{MPa}$ ).

To determine the optimal composition of solid dispersion, a mathematical planning method employing MathCad package and Excel software was applied. The experiment was designed using the least squares method (LSM) in the MathCad and Excel software. LSM is the basic method of regression analysis for estimating unknown parameters of regression models based on sample data, where the criterion of optimality is the minimum sum of squares of deviations of calculated values of Y from the experimental: min $\Sigma i$ (Ys, $\mathrm{i}-\mathrm{Yi})^{2}$. The minimum of the function is found by differentiating by parameters and is equal to zero. As a result, the search for a minimum sum of squares is reduced to simple operations with matrices [5]. The solubility was determined by utilizing the spectrophotometric method. The absorption spectra of quercetin solutions obtained were taken on Evolution 60-S spectrophotometer in a cell with layer thickness of $10 \mathrm{~mm}$. Determination of pharmaco-technological parameters was carried out in accordance with pharmacopoeiaspecified techniques [6].

\section{RESULTS}

To determine the rational composition, mathematical planning of the experiment was carried out to ascertain the influence of carrier and ethanol on the degree of quercetin solubility. Substances were grouped by two qualitative factors (Table. 1).

Table 1. Excipients that have been studied to improve the degree of dissolution

\begin{tabular}{|l|c|}
\hline \multicolumn{1}{|c|}{ Factors } & Factor levels \\
\hline A - carrier & a1 - PEO:Q 1:1, a2 - $\beta-$ CD: Q 1:1, a3 - PEO: Q 2:1, \\
& a4- - -CD: Q 2:1, a5 - PVP: Q 1:1, a6 - PVP: Q 2:1 \\
\hline B - solvent & b1 - with solvent \\
b2 - solvent free
\end{tabular}

After determining the factors and their levels, the experimental plan was selected (Table 2).

Table 2. Two-factor plan of the experiment and the results of the study on the degree of quercetin dissolution, $\%$

\begin{tabular}{|c|c|c|c|c|c|c|c|}
\hline \multirow{2}{*}{ Factor B } & \multicolumn{6}{|c|}{ Factor A } & \multirow{2}{*}{$\begin{array}{l}\text { Amount } \\
\text { of factor } \\
\text { levels B }\end{array}$} \\
\hline & $\mathrm{a} 1$ & a2 & a3 & a4 & a5 & a6 & \\
\hline \multirow{3}{*}{ b1 } & 0.84 & 0.58 & 0.71 & 0.5 & 0.63 & 0.55 & 3.81 \\
\hline & 0.88 & 0.66 & 0.75 & 0.48 & 0.6 & 0.54 & 3.91 \\
\hline & 0.95 & 0.67 & 0.72 & 0.52 & 0.62 & 0.52 & 4.00 \\
\hline \multirow{3}{*}{ b2 } & 5.38 & 3.85 & 6.72 & 2.8 & 1.1 & 0.95 & 20.80 \\
\hline & 5.52 & 3.79 & 6.68 & 2.83 & 0.95 & 1.05 & 20.82 \\
\hline & 5.43 & 3.8 & 6.83 & 2.81 & 0.94 & 0.99 & 20.80 \\
\hline $\begin{array}{l}\text { Amount of } \\
\text { factor levels A }\end{array}$ & 19.00 & 13.35 & 22.41 & 9.94 & 4.84 & 4.60 & 74.14 \\
\hline $\begin{array}{l}\text { Square } \\
\text { of amount }\end{array}$ & 361.00 & 178.23 & 502.21 & 98.80 & 23.43 & 21.16 & 5496.74 \\
\hline
\end{tabular}

The results of the dispersion analysis (Fig. 1) show that the presence of solvent essentially affects the degree of dissolution.

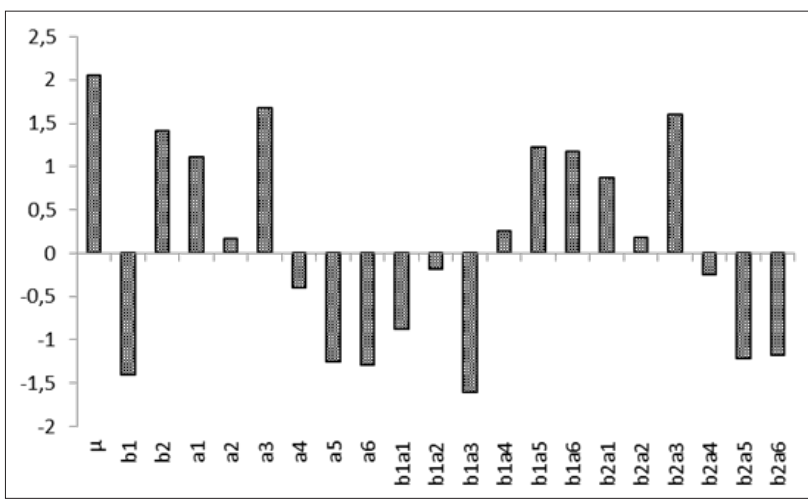

Figure 1. Graphic representation of the influence of factors

The Fischer criterion calculation ( $F$ - criterion) has revealed that the factor $B\left(F_{B} \exp ^{\cos }=28817, F_{B}{ }^{0.05}=4.26\right)$ and the factor $A\left(F_{A}^{\exp }=3614, F_{A}^{0.05}=2.62\right)$ are significant, but the interaction between the factors $A$ and $B$ does not significantly affect the process of dissolution of the solid dispersion $\left(F_{A B}{ }^{\exp }=3042, F_{A B}^{0.05}=2.62\right)$.

In general, impact on A factor of some of its levels decreases in the row $a 3>a 6>a 5>a 1>a 4>a 2$. When combining various levels of factors $\mathrm{A}$ and $\mathrm{B}$, the following sequence of influence is observed: $\mathrm{b} 1 \mathrm{a} 3>\mathrm{b} 2 \mathrm{a} 3>\mathrm{b} 2 \mathrm{a} 5=$ $\mathrm{b} 1 \mathrm{a} 5>\mathrm{b} 1 \mathrm{a} 6=\mathrm{b} 2 \mathrm{a} 6>\mathrm{b} 2 \mathrm{a} 1=\mathrm{b} 1 \mathrm{a} 1>\mathrm{b} 1 \mathrm{a} 4>\mathrm{b} 2 \mathrm{a} 4>\mathrm{b} 2 \mathrm{a} 2$ $=\mathrm{b} 1 \mathrm{a} 2$. Adding PEO-6000 in the ratio of 2: 1 has a significant effect, both with the addition of ethanol and without it. Inconsistency, however, affects the levels of factors and their combinations: a1, a2, a4, b1a1, b1a2, b1a4, b2a1, b2a2, b2a4.

Thus, the results of the dispersion analysis show that PEO-6000 presence has a significant effect on the degree of solubility. The next step was to determine the appropriate content of PEO-6000 in the solid dispersion. For the complex definition, the parameters of the obtained solid dispersion (bulk density, fluidity) and model specimens of tablets based on it (disintegration, mechanical strength and friability, dissolution time) were used as responses.

The factors and their levels studied when optimizing the solid dispersion's composition are given in Table 3. 
Table 3. Factors and their levels studied

\begin{tabular}{|l|c|}
\hline \multicolumn{1}{|c|}{ Factors } & Factor levels \\
\hline A - carrier & $\mathrm{a} 1-$ PEO \\
\hline D - ratio API/ carrier & $\mathrm{d} 1-1$ \\
& $\mathrm{~d} 2-0.5$ \\
\hline
\end{tabular}

The experiment planning matrix and the results of the study are presented in Table 4.

Table 4. Plan of the experiment to study the effect of PEO-6000 amounts on the properties of solid dispersion

\begin{tabular}{|c|c|c|c|c|c|c|c|}
\hline № & $\mathrm{D}$ & $\mathrm{Y} 1$ & $\mathrm{Y} 2$ & $\mathrm{Y} 3$ & $\mathrm{Y} 4$ & $\mathrm{Y} 5$ & $\mathrm{Y} 6$ \\
\hline 1 & $\mathrm{~d} 1$ & 180 & 0.86 & 6.7 & 184 & 0.95 & 5.44 \\
\hline 2 & $\mathrm{~d} 2$ & 270 & 0.79 & 8.1 & 195 & 0.87 & 5.33 \\
\hline 3 & $\mathrm{~d} 3$ & 318 & 0.81 & 9.5 & 198 & 0.47 & 5.15 \\
\hline
\end{tabular}

Notes: $y_{1}$ - time of disintegration, $s ; y_{2}-$ bulk density, $g / \mathrm{ml} ; y_{3}-$ flowability, $\mathrm{g} / \mathrm{s} ; \mathrm{Y}_{4}-$ mechanical strength, $\mathrm{N} ; \mathrm{Y}_{5}-$ friability, $\% ; \mathrm{Y}_{6}$ - amount of substance dissolved, \%

To study the effect of the filler amount on the properties of solid dispersion, corresponding matrices were then made up for further processing via applied software Mathcad according to the experimental plan:

$\mathrm{D}:=\left(\begin{array}{c}1 \\ 0.5 \\ 0.33\end{array}\right) \quad \mathrm{y} 1:=\left(\begin{array}{c}180 \\ 270 \\ 318\end{array}\right) \quad \mathrm{y} 2:=\left(\begin{array}{c}0.86 \\ 0.79 \\ 0.81\end{array}\right) \quad \mathrm{y} 3:=\left(\begin{array}{l}6.7 \\ 8.1 \\ 9.5\end{array}\right) \quad \mathrm{y} 4:=\left(\begin{array}{c}184 \\ 195 \\ 198\end{array}\right)$ y5 $:=\left(\begin{array}{c}0.95 \\ 0.87 \\ 0.47\end{array}\right) \quad \mathrm{y} 6:=\left(\begin{array}{c}5.44 \\ 5.33 \\ 5.15\end{array}\right)$

Graphic representations of experimental data of technological properties of solid dispersions are shown in Fig. 2-6.

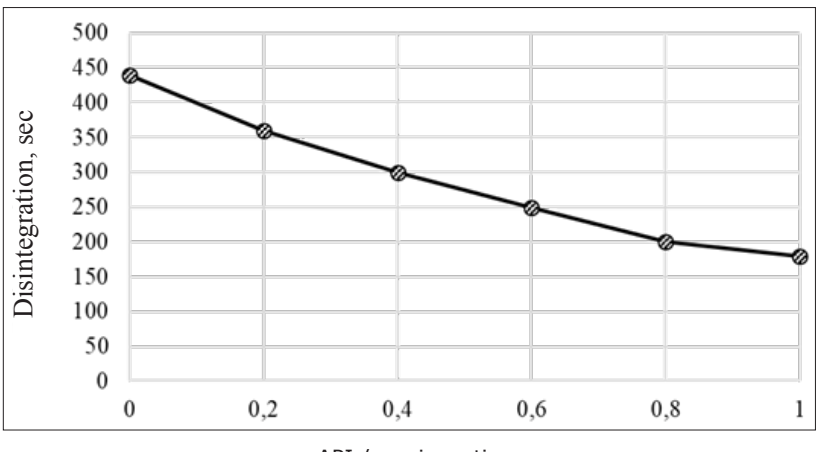

Figure 2. Graphical dependences of disintegration time on the ratio of PEO-6000 and quercetin model specimens of tablets

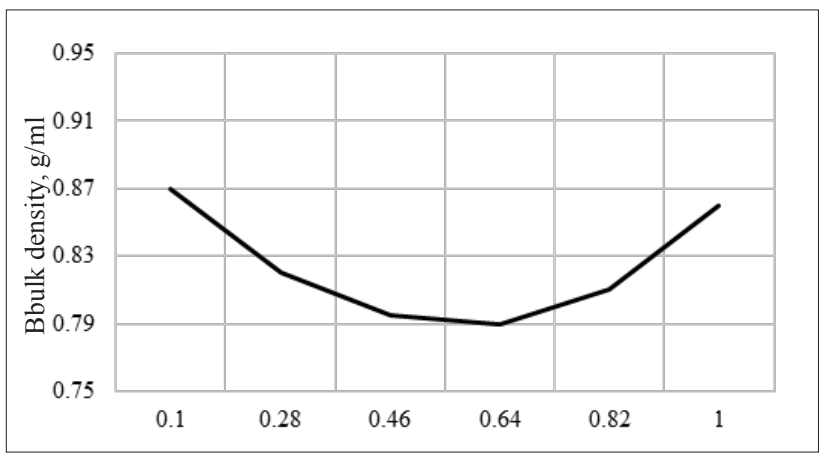

API / PEO-6000

Figure 3. Graphic dependences of bulk density on the ratio of PEO-6000 and quercetin solid dispersion

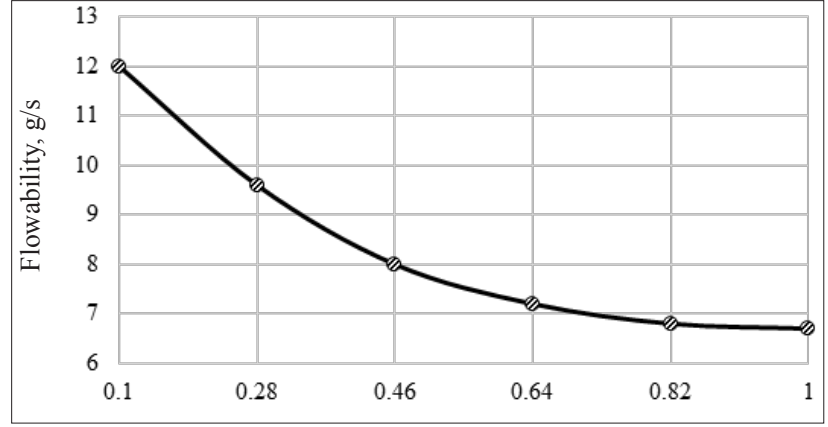

API / PEO-6000

Figure 4. Graphic dependences of flowability on the ratio of PEO-6000 and quercetin solid dispersion

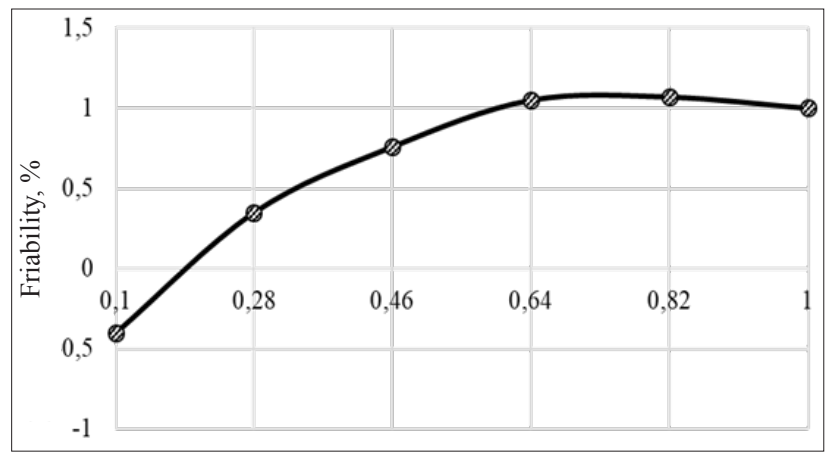

API / PEO-6000

Figure 5. Graphical dependences of friability on the ratio of PEO-6000 and quercetin model specimens of tablets

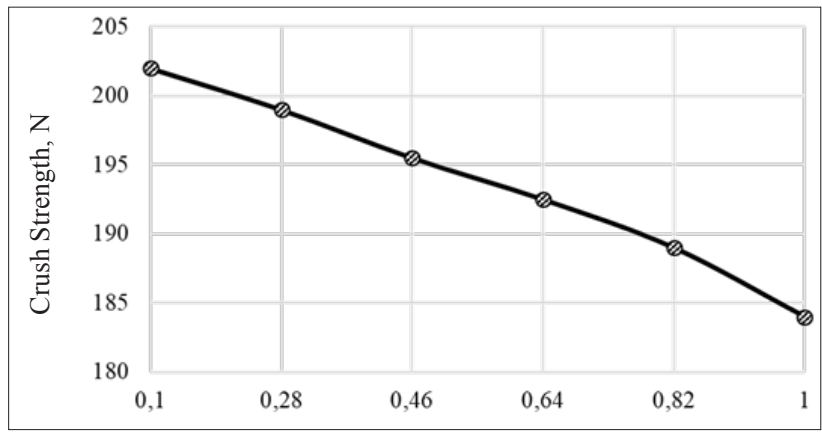

API / PEO-6000

Figure 6. Graphic dependences of mechanical strength on the ratio of PEO-6000 and quercetin model specimens of tablets

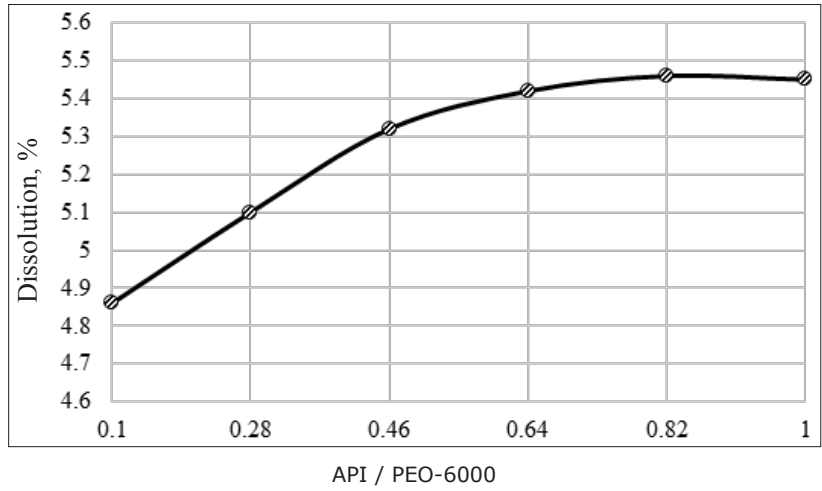

Figure 7. Graphic dependences of the degree of dissolution on the ratio of PEO-6000 and quercetin model specimens of tablets 
Empirical functional dependences were found with regard to the corresponding dependence of quality indicators on the ratio of quercetin and PEO-6000. These are given in Table 5.

Table 5. Regression equation

\begin{tabular}{|c|c|}
\hline Index & $Y=f(d)$ \\
\hline$y 1$ & $y 1(d)=436.383-409.1748 d+152.766 d^{2}$ \\
\hline$y 2$ & $y 2(d)=0.912-0.437 d+0.385 d^{2}$ \\
\hline$y 3$ & $y 3(d)=13.556-14.969 d+8.112 d^{2}$ \\
\hline$y 4$ & $y 4(d)=202.752-12.255 d-6.497 d^{2}$ \\
\hline$y 5$ & $y 5(d)=-0.847+5.07 d-3.273 d^{2}$ \\
\hline$y 6$ & $y 6(d)=4.594+2.098 d-1.252 d^{2}$ \\
\hline
\end{tabular}

The dependence of the pharmaco-technological indices of the solid dispersion on the ratio of API/carrier is determined by second order regression equations. The adequacy of the obtained equations is confirmed by the complete coincidence of theoretical calculations and experimental points. The error value calculated using Fisher's criterion was $0.5 \%$.

After the mathematical processing of the empirical dependencies $y i=f(d)$, we determined the amount of PEO- 6000 in the solid dispersion that would provide all the necessary technological parameters within the appropriate permissible values intervals, namely, disintegration time (y1) from 150 to $318 \mathrm{~s}$, the bulk density (y2) from 0.80 to $0.90 \mathrm{~g} / \mathrm{ml}$, flowability (y3) from 6,5 to $9.5 \mathrm{~g} / \mathrm{s}$, mechanical strength (y4) from 180 to $200 \mathrm{~N}$, friability of model specimens (y5) from 0.45 to $1.0 \%$ (y6) and dissolved amount from 4.5 to $5.7 \%$. Intervals of values were ascertained taking into account the results of experimental studies. To solve this problem, the theory of multi-vector optimization was applied, according to which the system of the obtained equations, together with the imposed restrictions on the values of functions and the variable, has an area of positive solutions (Fig. 8).

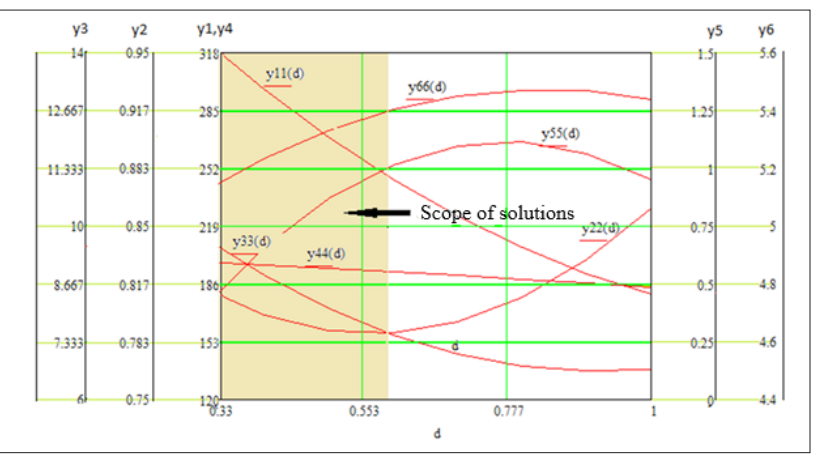

Figure 8. Scope of possible solutions: 1 - the value of the disintegration time, $\mathrm{s} ; 2$ - bulk density, $\mathrm{g} / \mathrm{ml} ; 3$ - flowability, g/s; 4 - mechanical strength, N; 5 - friability, \%; 6 - amount dissolved, \%

From this multitude, we selected the value of the variable that approximated all the technological parameters to the desired values in an equal measure. The solution was found based on the condition that the sum of squared deviations of each indicator from the given value will be minimal [5].

When minimizing the vector function $R(d)=f(y 1(d)$, y2 (d), y3 (d), y4 (d), y5 (d), y6 (d)), the optimal values for the ratio of quercetin and PEO- 6000 were obtained. These are $1: 2$.

\section{CONCLUSIONS}

To determine the rational composition of the solid dispersion of quercetin, mathematical planning of an experiment on optimization of its composition was carried out in two stages by means of a two-factor experiment based on the Graeco-Latin square: the determination of the optimal carrier and its amount. A dispersion analysis was then performed according to the results of experimental studies. The ranked series of advantages of carrier and solvent influence on solubility of solid dispersions samples are shown.

As the response used the dissolution degree of solid dispersion samples, to determine the qualitative composition, the excipients were grouped together by two factors. The results of the dispersion analysis reveal that the presence of PEO-6000 has a significant effect on the degree of solubility.

In order to determine the best ratio of carrier and API in the solid dispersion, bulk density, SD powder flowability, disintegration time, dissolution time, friability and mechanical strength for the model tablets were chosen as suitable responses. We then established that the dependence of disintegration time, bulk density, strength, solubility on the ratio of PEO-6000 and quercetin is determined by second-order regression equation. A dispersion analysis was subsequently performed to ascertain the amount of carrier in the solid dispersion. According to the results of the conducted studies, the optimal value ration of quercetin and PEO-6000 is $1: 2$.

The obtained results can be used in the development of the composition and technology of solid dispersions with these excipients, as well as active pharmaceutical ingredients with similar physicochemical properties that are insoluble in aqueous medium.

\section{ORCID iDs}

Inna Kovalevska (Dhttps://orcid.org/0000-0001-5610-8334 Olena Ruban (1)https://orcid.org/0000-0002-2456-8210 Olga Kutova (1) https://orcid.org/0000-0002-3761-2831 Julia Levachkova (Dhttps://orcid.org/0000-0002-8540-4041

\section{REFERENCE}

1. Bajaj S, Khan A. Antioxidants and diabetes. IJEM. 2012;16:267-71.

2. Ioku K, Tsushida T, Takei Y, Nakatani N, Terao J. Antioxidative activity of quercetin and quercetin monoglucosides in solution and phospholipid bilayers. Biochim Biophys Acta. 1995;1234:99-104.

3. Kovalevska IV, Ruban EA, Kutsenko SA, Kutova OV, Kovalenko Sv. Study of physical and chemical properties of solid dispersions of quercetin. Asian J Pharm. 2017;11(4):805-9.

4. Kovalevska I, Ruban O. Development of the methodological approach of obtaining preparations based on solid dispersions. Sci Rise Pharm. 2018;4(14):4-8.

5. Kutova OV, Kovalevska IV, Kutovyi DS, Shapovalov OV, Zhuravsky AO. Certificate of registration of copyright to work 83778 Ukraine. Literary piece of writing scientific character Method of determining the optimal parameters of the process. Date of registration 18.12.2018.

6. State Pharmacopoeia of Ukraine. In State Enterprise «Ukrainian Scientific Pharmacopoeial Center for the Quality of Medicines» Vol. 3 -2 ed. Completed 1 - Kharkiv: State Enterprise. Ukrainian Scientific Pharmacopoeia Center for Quality of Medicinal Products. 2015;1128. 